\title{
Diffuse large B cell lymphoma (DLBCL) in patients older than 65 years: analysis of 3 year Real World data of practice patterns and outcomes in England
}

L. Hounsome ${ }^{1,13}$, T. A. Eyre (DD ${ }^{2,13}$, R. Ireland ${ }^{3}$, A. Hodson ${ }^{4}$, R. Walewska ${ }^{5}$, K. Ardeshna ${ }^{6}$, S. Chaganti ${ }^{7}$, P. McKay ${ }^{8}$, A. Davies ${ }^{9}$, C. P. Fox ${ }^{10}$, N. Kalakonda ${ }^{11}$ and P. A. Fields (iD ${ }^{12}$

(c) The Author(s) 2021

BACKGROUND: We wished to examine treatment and outcome patterns in older diffuse large B-cell lymphoma (DLBCL) patients, with a focus on the effect of route-to-diagnosis to outcome.

METHODS: Data were extracted from Public Health England's National Cancer Registration and Analysis Service between 2013 and 2015 included route-to-diagnosis, disease characteristics and survival for 9186 patients $\geq 65$ years. Systemic Anti-Cancer Therapy data identified front-line regimens, cycles and doses.

RESULTS: Route-to-diagnosis were emergency (34\%), NHS urgent cancer pathway (rapid haemato-oncologist review <2 weeks), (29\%) and standard GP referral (25\%). The most common regimen was R-CHOP $(n=4392) .313$ patients received R-miniCHOP $(7 \%$ of R-CHOP). For all patients, 3-year overall survival (OS) for 65-79 years was $57 \%$ and for $\geq 80$ years was $32 \%$. Three-year OS for RCHOP-treated patients diagnosed via emergency presentation was 54\% (adjusted hazard ratio (HR) $1.63, p<0.01$ ) and $75 \%$ (adjusted HR 0.81, $p<0.01$ ) on the NHS urgent cancer pathway (reference HR:1.00: GP referrals). 3-year OS was 54\% for both $\mathrm{R}$-miniCHOP and $\mathrm{R}-\mathrm{CHOP}$ in $\geq 80$ years.

CONCLUSIONS: Our comprehensive population analysis is the first to show that the NHS urgent cancer pathway is associated with a superior survival after adjusting for multiple confounders. Equivalent survival for R-CHOP and R-mini-CHOP was demonstrated in those $\geq 80$ years.

British Journal of Cancer (2022) 126:134-143; https://doi.org/10.1038/s41416-021-01525-4

\section{INTRODUCTION}

Diffuse large B-cell lymphoma (DLBCL) is the most common B cell non-Hodgkin lymphoma (NHL) [1]. Incidence increases with age $[2,3]$ and presentation is most common in patients over 65 years (https://www.hmrn.org/statistics/incidence). The majority are treated outside of prospective trials with treatment selected by their supervising haemato-oncologists. There is limited population-level data on referral and treatment patterns and patient outcomes. In the UK, the NHS Cancer Plan in the year 2000 (https://webarchive. nationalarchives.gov.uk/20130123203940/, http://www.dh.gov.uk/ en/Publicationsandstatistics/Publications/PublicationsPolicyAnd Guidance/DH_4010198) [4] announced the NHS urgent cancer referral pathway - the "Two Week Wait" diagnosis pathway initiative the implementation for all suspected cancer patients. This national pathway was developed to expedite an urgent specialist referral from general practice (the GP mandates the patient is seen within 2 weeks by a specialist) for patients whose signs or symptoms were suggestive of cancer. The aim is to enable early diagnostic work up under a specialist, diagnosis and improve outcome. Although clinical trial outcomes are well documented, the outcomes of DLBCL patients treated in a real-world setting across the whole population of England are not described. Specifically, the effect on outcomes by the route to diagnosis, treatment regimen choice, comorbidity and deprivation quintile, have not been described.

DLBCL commonly presents in patients with comorbidities or the very elderly (e.g. $\geq 80$ years). Many of these patients receive R-CHOP (rituximab, cyclophosphamide, doxorubicin, vincristine and prednisolone) or R-CHOP-like therapy based on fitness. To date, no randomised trials comparing cyclophosphamide and doxorubicin dose(s) in elderly DLBCL patients have been performed. Attenuated 'R-miniCHOP' is now widely accepted as

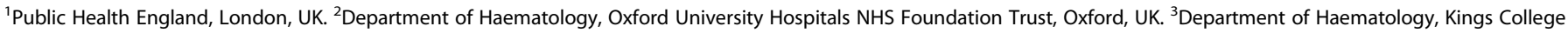

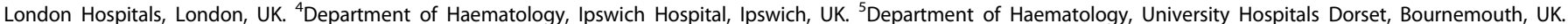

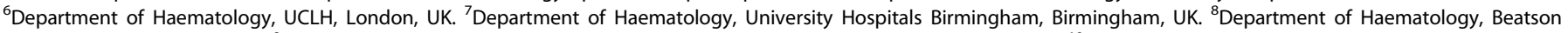

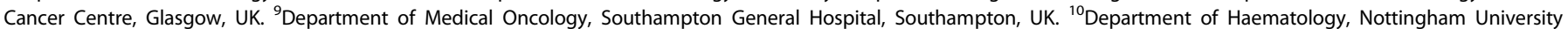

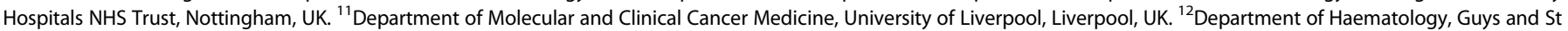
Thomas' Hospital, London, UK. ${ }^{13}$ These authors contributed equally: Hounsome L, Eyre TA. ${ }^{\circledR}$ email: paul.fields@gstt.nhs.uk
} 
a standard approach based on the LYSA phase II trial which demonstrated that R-miniCHOP (doxorubicin $25 \mathrm{mg} / \mathrm{m}^{2}$, cyclophosphamide $400 \mathrm{mg} / \mathrm{m}^{2}$ and vincristine $1 \mathrm{mg}$ capped-dose) provided curative potential in patients $\geq 80$ years [5] with a 2-year PFS and 2 -years OS of $47 \%$ and $59 \%$, respectively. In light of this study and a subsequent trial of ofatumumab-miniCHOP [6], 'R-miniCHOP' has become widely adopted. The question of dose intensity of R-CHOP that addresses disease control and toxicity risk in elderly individuals remains unresolved, with little prospective data to guide decision making. Available retrospective series suggest that in patients $\geq 80$ years, [7-9] there is minimal evidence to support full dose R-CHOP for all patients considered fit for anthracyclinebased immunochemotherapy. R-miniCHOP has recently been favoured as the standard arm of the Lymphoma Study Association (LYSA) randomised SENIOR trial in patients $\geq 80$ years [10].

To examine these factors, we sourced data from the National Cancer Registration and Analysis Service (NCRAS), part of Public Health England (PHE). NCRAS maintains the only wholepopulation comprehensive and mandatory registry of cancer diagnoses in England (https://www.gov.uk/guidance/nationalcancer-registration-and-analysis-serviceNCRAS). The registry holds detailed data on each tumour type and is linked to other clinical information such as the Systemic Anti-Cancer Therapy (SACT) dataset. This dataset holds SACT activity from all NHS England providers, sourced from e-prescribing systems [11]. We wished to analyse characteristics and survival outcomes for the DLBCL population in England aged $\geq 65$ years registered in NCRAS (defined by International Classification of Diseases-10 (ICD10) code C83.3). We hypothesised that patients diagnosed and treated following an NHS urgent cancer pathway referral would have improved survival.

\section{METHODS}

Data were obtained from PHE NCRAS via a formal application to the organisation's PHE's Office for Data Release (ODR). Data were assessed as suitable for release without specific ethical permissions by ODR (ODR assignment no:1617_136) and the NCRAS Caldicott Guardian. All DLBCL diagnoses (ICD10 C83.3) in patients $\geq 65$ years in England in 2013-2015 were identified in NCRAS. At date of extraction, 2013-2015 was the most recent period for which full three-year follow-up of vital status was available. Data were collected for route to diagnosis, extracted on age, sex, ethnicity, stage, comorbidity (modified Charlson Comorbidity index), deprivation index, vital status, and cause of death. The deprivation measure was the Income domain of English Indices of Deprivation 2010 (see supplement for further details) (https://www.gov.uk/government/ statistics/english-indices-of-deprivation-2010). The route to diagnosis is a derived measure of the way in which a patient arrived at their cancer diagnosis, based on a combination of several datasets [12]. Data on frontline chemotherapy was obtained from SACT and included regimens, cycle dates, treatment intent, and regimen modifications (e.g. RminiCHOP). All relevant comorbid conditions were assigned a weight from 1 to 6 , based on specific diagnosis codes found in hospital admissions in a 3-27 month period prior to DLBCL diagnosis. These weights were then summated to produce an abbreviated comorbidity index. Fig. S1 provides a summary of the methodology and timelines of accessing and linking relevant data.

Data were linked and analysed using Stata 15 (StataCorp. 2017. College Station, TX: StataCorp LLC). Kaplan-Meier survival curves were generated using STS command. Overall survival (OS) was defined from DLBCL diagnosis until time of death from any cause and differences in OS were determined by log-rank tests. Lymphoma-specific survival (LSS) was defined from DLBCL diagnosis until the time of death from DLBCL, with censoring occurring at the cause of death for other unrelated causes other than DLBCL. To account for the impact of multiple variables on survival, a Cox-regression model was implemented. Differences in proportions for descriptive statistics were tested with a 2-sided Z-test. Data were interrogated to explore potential associations between route to diagnosis and baseline characteristics with OS and LSS. We also evaluated R-CHOP intended dose intensity (IDI) across the population-with a particular focus on very elderly ( $\geq 80$ years). Patients were analysed according to categories
$65-79$ and $\geq 80$ years as dose intensity decisions typically vary according to those age cut-offs. IDI was determined by strict first cycle total dosage analysis of individual drug components (defined for R-miniCHOP: vincristine $1 \mathrm{mg}$, doxorubicin $\leq 55 \mathrm{mg}$, and cyclophosphamide $\leq 880 \mathrm{mg}$ ). These thresholds were chosen to include all patients receiving R-miniCHOP up to a maximum body surface area of $2.2 \mathrm{~m}^{2}$ based on the standard R-miniCHOP doses $\left(400 \mathrm{mg} / \mathrm{m}^{2}\right.$ cyclophosphamide, $25 \mathrm{mg} / \mathrm{m}^{2}$ doxorubicin). R-CHOP was defined as either cyclophosphamide or doxorubicin doses greater than R-miniCHOP defined thresholds. The study was performed in accordance with the Declaration of Helsinki. All authors had full access to the data and the corresponding author had final responsibility for the decision to submit the manuscript for publication.

\section{RESULTS}

A total of 9186 DLBCL patients $\geq 65$ years across a 3 -year period $(01 / 01 / 2013-31 / 12 / 2015)$ were identified in the NCRAS database. Table 1 describes key baseline characteristics. Median follow-up of the whole cohort was 2.1 years. The cohort was largely aged $65-79$ years $(n=6203,68 \%)$. Gender distribution was broadly equal. Less deprived population quintiles (quintiles 1-2) had numerically more cases. Men were more likely to be younger $(55 \%$ of those $65-79$ years vs $49 \%$ of those $\geq 80$ years; $p<0.001$ ) and with lower levels of deprivation (56\% in quintile 1 vs $51 \%$ in quintile $5 ; p=0.008)$. Across all patients, 4536 presented with stage III-IV (61\% of available data) and 2918 presented with earlystage DLBCL (39\%). The proportion diagnosed with advanced stage decreased with increasing age $(63 \%$ in $65-79$ years vs $56 \%$ in $\geq 80$ years; $p<0.001)$. Characteristics according to year were similar (data not shown)

\section{Route to diagnosis}

Across all patients, the most common route to diagnosis was emergency presentation (35\%). A substantial proportion also presented via the NHS urgent cancer pathway referral (29\%) and a standard elective GP referral (timed 'two week wait pathway' not mandated) (25\%). Nine percent of patients were referred from other outpatient services. Emergency presentation was more common in advanced stage disease; of those presenting via emergency route, $69 \%$ had stage III-IV disease. In contrast, the most common route to diagnosis for early-stage disease was via the NHS urgent cancer referral pathway. There were no differences in emergency presentation proportion by sex, however, emergency presentations were more common in older patients $(37 \%$ in $\geq 80$ years vs $33 \%$ in $65-79$ years; $p<0.001$ ). Baseline characteristics were available for 4392 R-CHOP-treated patients and are described in Table S1. Again, R-CHOP patients presenting as an emergency were significantly more likely to present with advanced stage disease compared to GP referrals or NHS urgent cancer referral pathway. Other demographics were relatively well matched across the diagnostic routes.

\section{Treatment regimen}

Of patients with a recorded regimen, the most common was R-CHOP ( $n=4392,48 \%$ ), followed by R-CVP (rituximab, cyclophosphamide, vincristine, prednisolone) (6\%), R-CEOP (rituximab, cyclophosphamide, etoposide vincristine, prednisolone) (2\%), R-PMITCEBO (1\%), R-EPOCH (rituximab, etoposide, prednisolone vincristine, cyclophosphamide, doxorubicin) (0.5\%) and R-GCVP (rituximab, gemcitabine, cyclophosphamide, vincristine, prednisolone) $(0.5 \%)$. Only $2 \%$ received first-line therapy within an interventional clinical study protocol. Approximately $40 \%$ had either no SACT regimen recorded or had another treatment outside of those described. Within that group, 1226 patients (13.3\%) received 'chemotherapy of unspecified type' and 599 patients $(6.5 \%)$ received radiotherapy alone and 814 patients (8.9\%) were known to receive palliative care support alone. Overall, R-CHOP represents $80.5 \%(4392 / 5453)$ of all DLBCL cases 
Table 1. Baseline characteristics according to age.

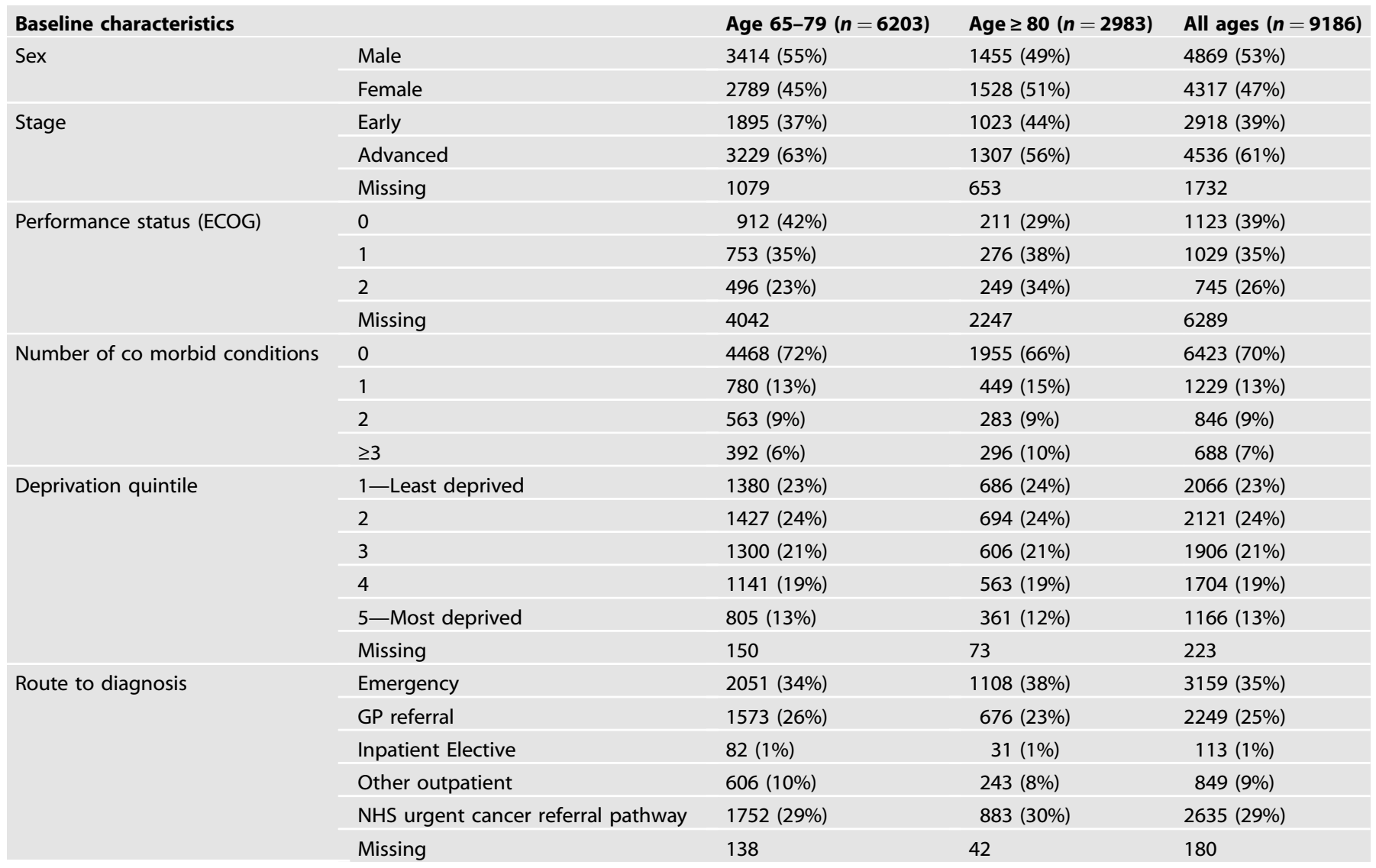

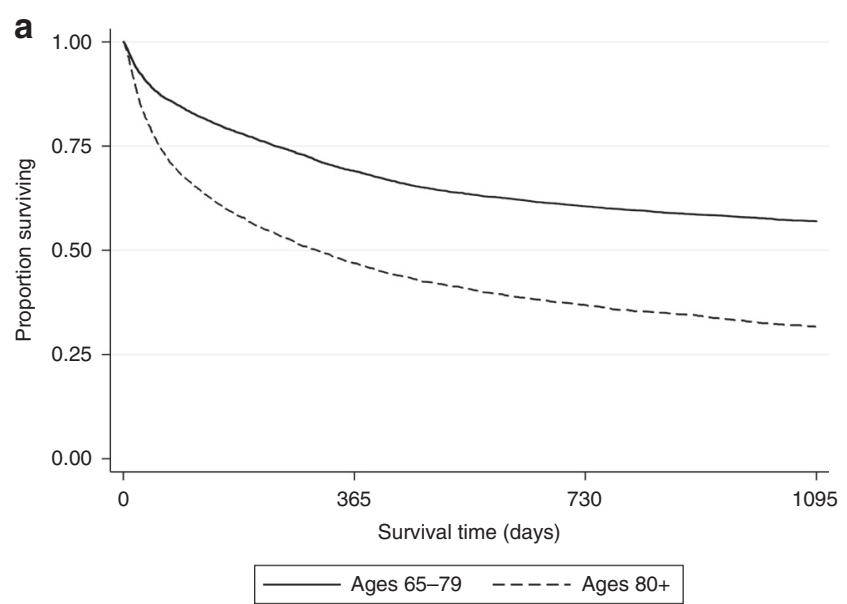

b

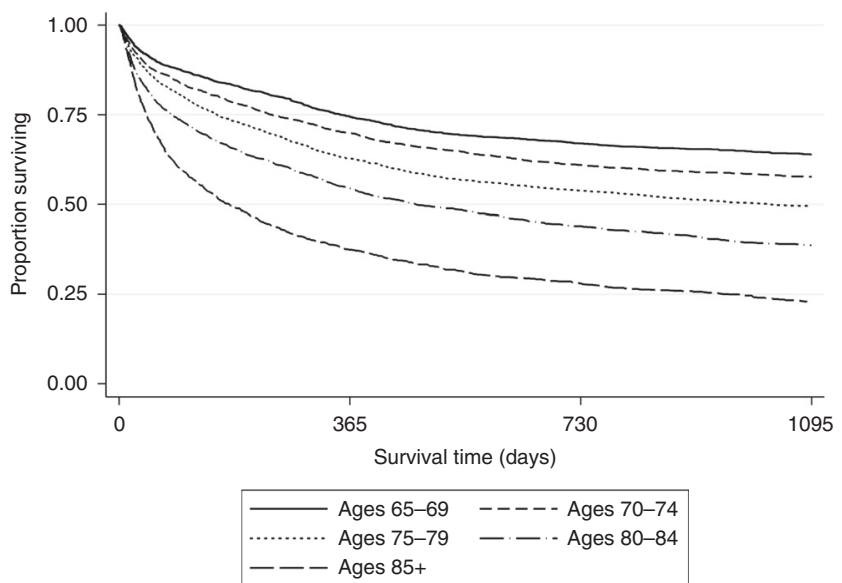

Fig. 1 Overall survival all DLBCL patients by age. a age $65-79$ vs $\geq 80$ years, $\mathbf{b}$ ages in 5 -year subgroups.

with a known frontline regimen. Trials listed in SACT were INCA [13], PHOENIX [14], and REMODL-B [15].

\section{Survival outcomes}

OS outcomes for all 9186 patients are shown in Fig. 1. 4819 (52\%) had died; with 1047 (22\%) of these recorded as due to DLBCL. Three-year OS was 49\% (95\% Cl 48-50\%). Three-year OS for those aged $65-79$ years was higher at $57 \%(95 \% \mathrm{Cl} 56-58 \%)$ than for those aged $\geq 80$ years at $32 \%(95 \% \mathrm{Cl} 30-33 \%)(p<0.001)$ (Fig. $1 \mathrm{a})$.
OS progressively worsened with advancing age (Fig. 1b). Threeyear OS for $65-69,70-74,75-79,80-84$ and $\geq 85$ years were: $64 \%$, $58 \%, 50 \%, 39 \%$ and $23 \%$, respectively ( $p<0.001$ for trend). Threeyear OS was $65 \%$ for patients receiving the regimens listed, $44 \%$ for all other regimens and $26 \%$ when no treatment was recorded by SACT $(p<0.001)$. 3-year OS for the NHS urgent cancer referral pathway was $74 \%(95 \% \mathrm{Cl} 72-76 \%)$, for GP referrals $68 \%(95 \% \mathrm{Cl}$ $66-71 \%)$, for other outpatients $68 \%(95 \% \mathrm{Cl} 63-73 \%)$ and for emergency presentation 53\% (95\% Cl 50-56\%). Survival according 
Table 2. Cox-regression analysis for overall survival for all R-CHOP treated patients.

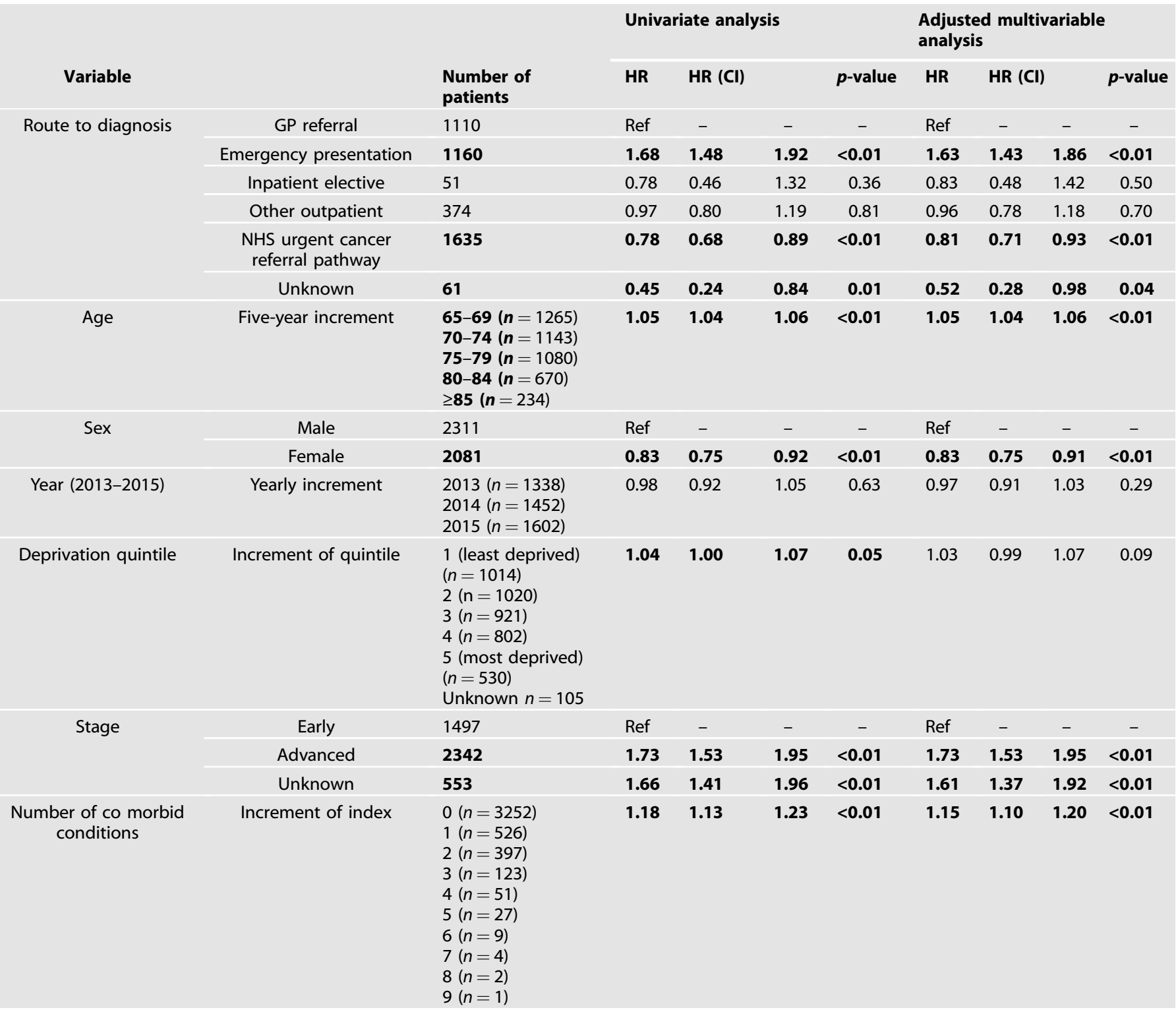

$H R$ hazard ratio, $\mathrm{Cl}$ confidence intervals at $95 \%$.

Statistically significant $p$-values are in bold.

to stage and route of presentation (Fig. 2S) shows that patients who have both early or late-stage DLBCL presenting as an emergency have an inferior OS compared with any other subgroup.

\section{Univariable (UVA) and multivariable survival analysis (MVA)}

Route of presentation. Table 2 presents the OS univariable (UVA) and multivariable (MVA) Cox-regression analysis for 4392 R-CHOPtreated patients. The 1635 patients who presented via the NHS urgent cancer referral pathway had a $22 \%$ lower risk of death on UVA compared to those presenting via routine elective GP referral $(p<0.01)$. This significant effect was retained on MVA (hazard ratio (HR) $0.81,95 \% \mathrm{Cl} 0.71-0.93, p<0.01$ ). In contrast, 1160 patients presenting via the emergency route displayed a $63 \%$ increased risk of death on MVA (HR 1.63,95\% Cl 1.43-1.86, $p<0.01$ ).

These findings were similar across the whole population. Figure 2 shows outcomes according to age and route of presentation in all patients. Three-year OS was superior for patients referred by the NHS urgent cancer referral pathway (65-79 years: $73 \%, \geq 80$ years: $44 \%$ ) and inferior by the emergency route (65-79 years: $39 \%$, $\geq 80$ years: $15 \%$ ) (Fig. 2a, b)

Other baseline factors. According to MVA, each 5-year age increment led to a $5 \%$ increased risk of death. Female patients had a $17 \%$ reduced risk of death compared to men. In an adjusted model, patients with late stage disease were at a $73 \%$ increased risk of death when compared to early stage. Patients with an higher comorbidity burden were also at increased risk of death, with each increment of comorbidity increasing risk by $15 \%$. Overall, patients with late stage disease, increased deprivation, male gender, and increased age (by 5 -year increment) all had an independently inferior survival. The results from an adjusted Coxregression analysis for LSS were similar. The notable difference was that comorbidity index became insignificant as a predictor of LSS (HR 0.97, 95\% 0.87-1.08, $p=0.60$ ). Importantly, the NHS urgent cancer referral pathway retained its association with an 


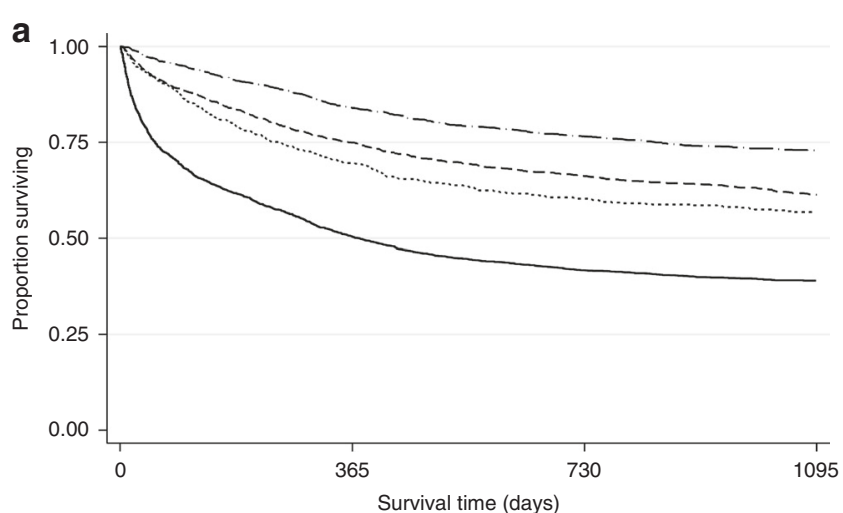

Emergency presentation $\quad-----$ GP referral Other outpatient $\quad$ - - - Two week wait
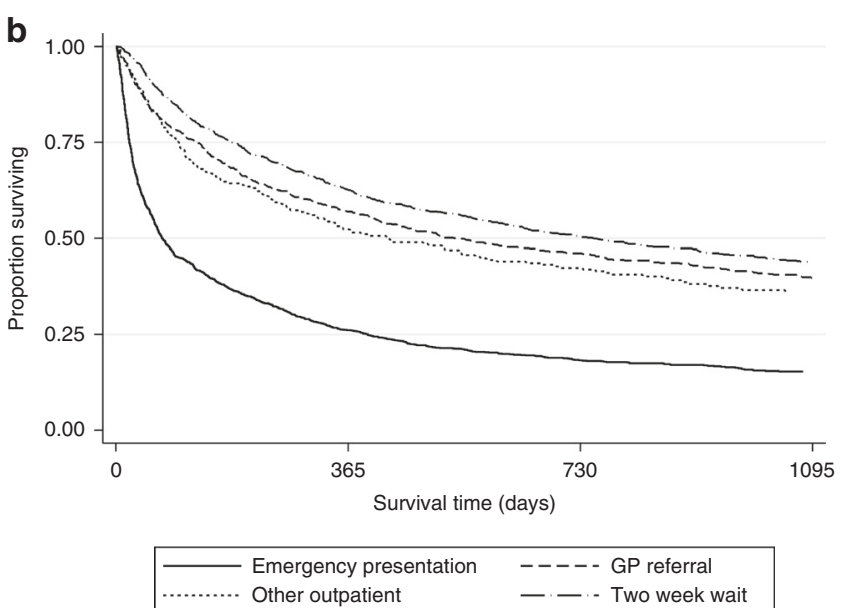

Fig. 2 Overall survival all patients DLBCL by route to diagnosis by age. a age 65-79 years (b) age $\geq 80$ years.

a

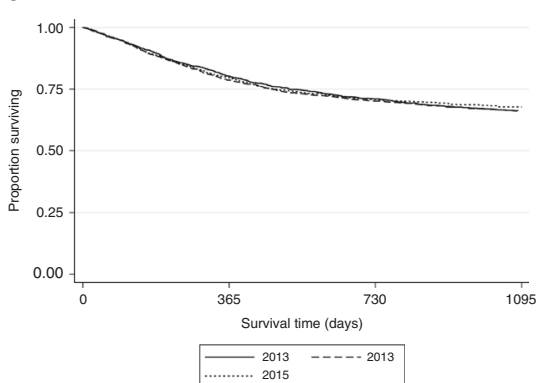

d

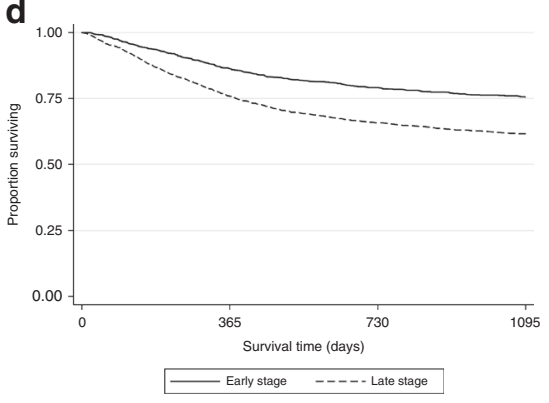

b

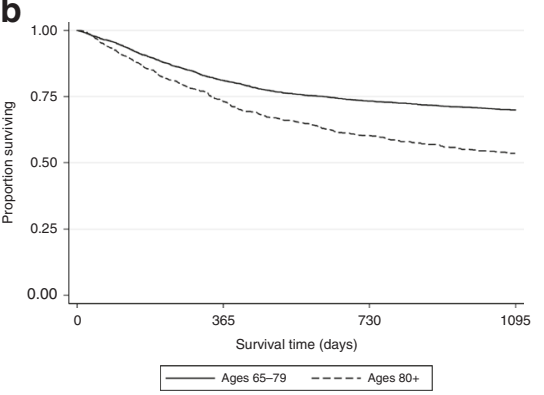

e

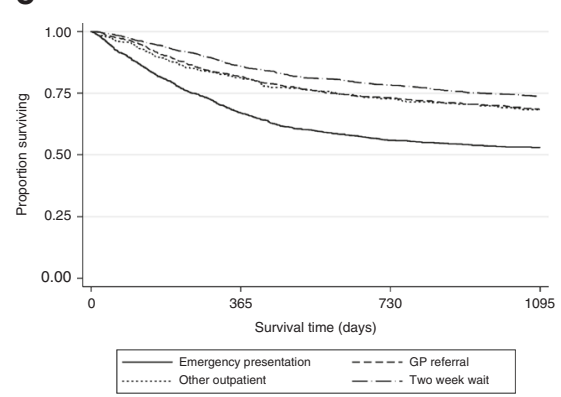

C

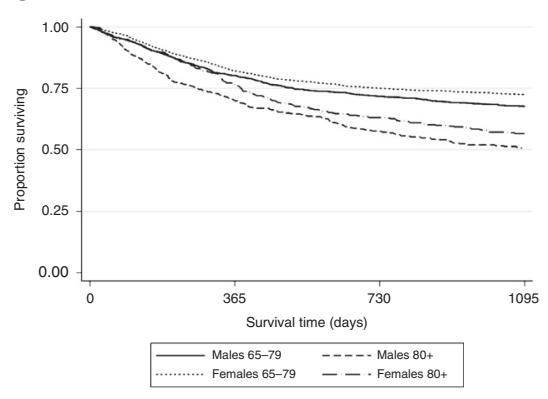

f

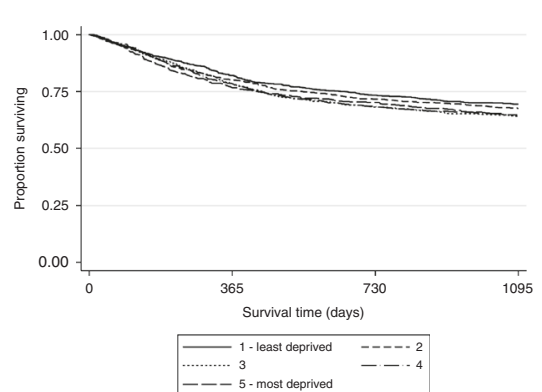

Fig. 3 Overall survival of all R-CHOP treated patients by. a year of diagnosis (b) by age $65-79, \geq 80$ years (c) age and sex (d) stage at diagnosis (e) route to diagnosis (f) deprivation.

improved LSS (HR 0.71, 95\% Cl 0.53-0.95, $p=0.02$ ), whilst patients with an emergency presentation had a significantly worse LSS by MVA (HR 1.95, 95\% Cl 1.49-2.54, p<0.01) (Table S2).

\section{Outcomes according to treatment regimen}

$R-C H O P .4392$ patients received $\mathrm{R}$-CHOP at either full or 'attenuated' dosing including 4079 patients receiving $\mathrm{R}-\mathrm{CHOP}$ at dosing greater than R-miniCHOP. Three-year OS was $68 \%(95 \% \mathrm{Cl}$ $66-69 \%)$ and did not differ across years analysed (Fig. 3a) $(p=$ $0.82)$. When analysed by age, 3-year OS was $70 \%$ (95\% Cl 69-72\%) for $65-79$ years and $54 \%$ (95\% Cl 49-57\%) for $\geq 80$ years (Fig. 3b) $(p<0.001)$. When analysed by age and sex, the highest three-year OS was observed in females aged 65-79 years (3-year OS 73\%) and lowest OS for men $\geq 80$ years (3-year OS 51\%) (Fig. 3c) $(p<$ $0.001)$. For patients with advanced stage disease, three-year OS was $62 \%(95 \% \mathrm{Cl} 60-64 \%)$ and for early-stage disease $77 \%(95 \% \mathrm{Cl}$ $75-79 \%)$ (Fig. 3d) ( $p<0.001)$. A significantly inferior OS was seen in patients with emergency presentation: 3-year OS $54 \%(95 \% \mathrm{Cl}$
$51-57 \%)$, and the highest observed OS of $75 \%$ (95\% Cl $72-77 \%)$ for the NHS urgent cancer referral pathway group (Fig. 3e) $(p<$ 0.001). Diagnosis to treatment interval was assessed in 3532 R-CHOP treated patients where data were available. DTI of 0-29 days was strongly associated with statistically shorter OS compared to DTI of $30+$ days ( $p<0.001$ ) (Fig. 3Sa). DTI was shorter in patients with emergency presentation (0-14 days $35.4 \%$, 15-29 days $34.8 \%$, chi-squared $p<0.001$ ) compared to GP referrals. NHS urgent cancer referral pathway DTI were statistically longer than GP referrals (Urgent NHS referral: 0-14 days 9.7\%, $15-29$ days $32.5 \%$, GP referral: $0-14$ days $13.5 \%, 15-29$ days $28.7 \%$, chi-squared $p<0.001$ ) (Fig. 3Sb).

There was a statistically significantly lower OS in more deprived groups $(p=0.01)$. The effect size, however, was small: 3-year OS in least deprived was $69 \%$ compared to $65 \%$ in most deprived (Fig. $3 f$ ).

R-miniCHOP. Of the R-CHOP-treated patients, 313 patients (7\%)) received R-miniCHOP. Three-year OS for the R-miniCHOP group 


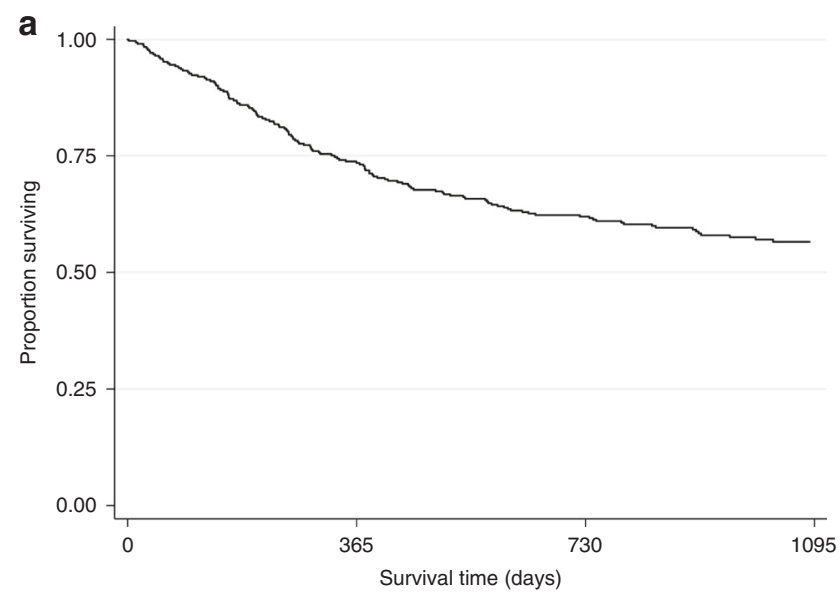

b
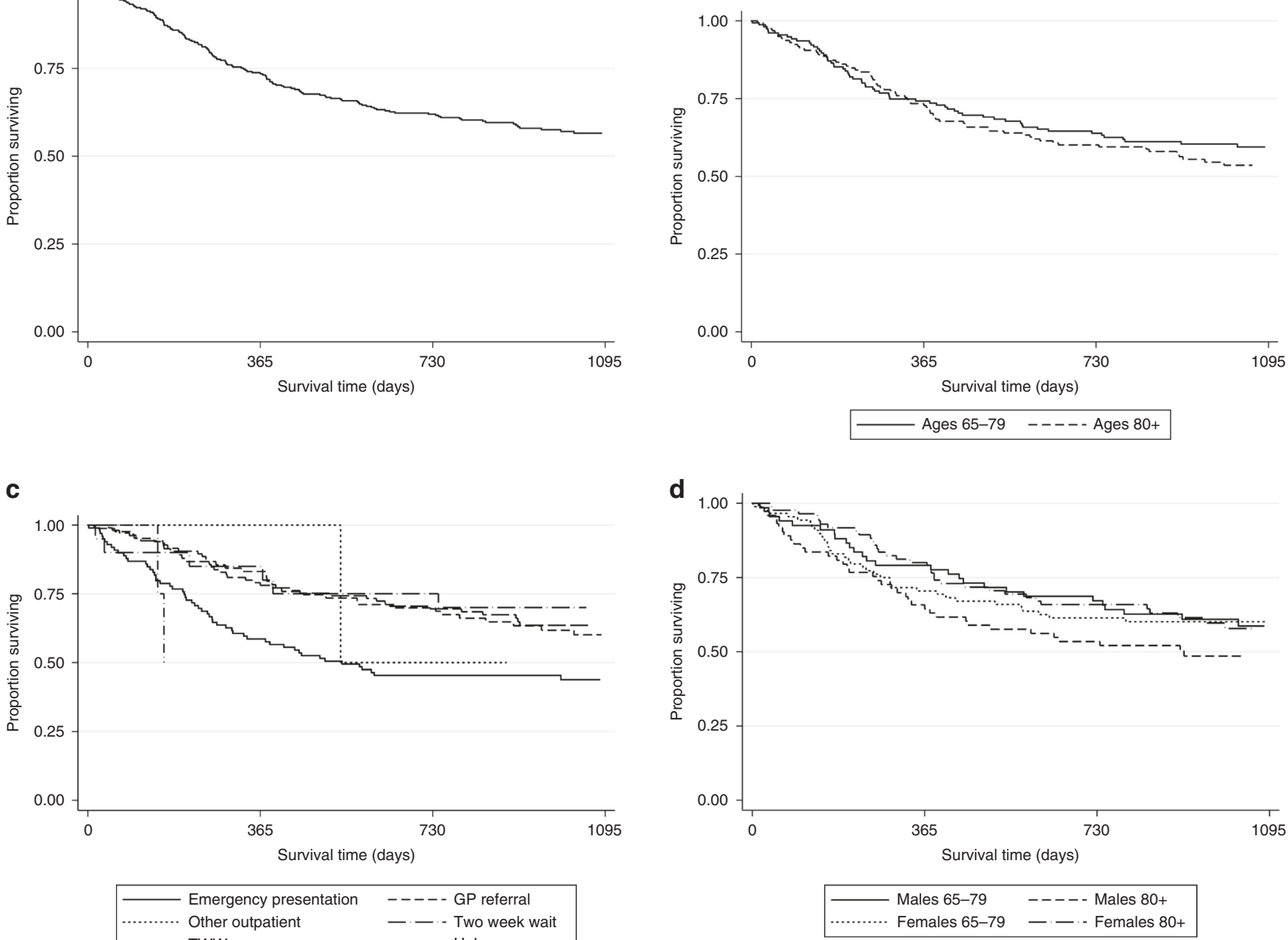

Fig. 4 Overall survival R-miniCHOP treated patients by. a all patients (b) by age $65-79, \geq 80$ years (c) route to diagnosis (d) age and sex.

was 57\% (95\% Cl 51\%-62\%) (Fig. 4a). Patients 65-79 years who received R-miniCHOP had a numerically higher comorbidity burden compared to those receiving standard R-CHOP (comorbidity index $\geq 1: 25 \% \mathrm{R}$-CHOP versus $35 \%$ for R-miniCHOP). When stratified by age (Fig. 4b), the 65-79 years group demonstrated a 3 -year OS of $59 \%$ (95\% Cl $51-67 \%)$ and $\geq 80$ years group $54 \%$ (95\% $\mathrm{Cl} 45-61 \%)$. Patients presenting via the emergency route had an inferior outcome as did males $\geq 80$ years (Fig. 4c, d). Two-year OS for patients $\geq 80$ years was $60 \%$ (95\% Cl $52-67 \%)$.

$R$-CHOP versus $R$-miniCHOP in patients $\geq 80$ years. Table 3 describes the characteristics of patients $\geq 80$ years divided by dose intensity. Proportionally more patients $\geq 85$ years received RminiCHOP, but otherwise the cohorts were strikingly well balanced according to stage, comorbidity, gender and deprivation index. Table S3 describes cycles received in patients $\geq 80$ years by dose intensity. Despite equivalent staging, patients receiving R-miniCHOP had a numerically higher proportion of patients who received 6-8 cycles compared to R-CHOP (54 vs 43\%) and less patients who received only $1-3$ cycles ( 29 vs $38 \%$ ) which may reflect disease stage. Figure 5 shows the OS of patients $\geq 80$ years treated with R-CHOP ( $n$ $=746$ ) or R-miniCHOP ( $n=158)$. OS curves completely overlap, with the 3-year OS for both groups equalling 54\%. There was no difference in LSS for the 2 groups either (Fig. 4S). When 73 patients $\geq 80$ years receiving full dose R-CHOP were compared to the 202 patients $\geq 80$ years receiving $\geq 1$ chemotherapeutic at full dose (but not all chemotherapeutics), there was no statistically significant difference in OS ((full dose 3-year OS $60.1 \%$ (Cl 48.9-74.0\%) vs. at least 1 chemotherapeutic at full dose 3-year OS $53.4 \% \quad(\mathrm{Cl}$ 46.8-60.9\%)). Regimen intensity (R-CHOP or R-miniCHOP) was included with an adjusted MVA of patients exclusively $\geq 80$ years (Table S4). Whilst late-stage disease (HR 1.31, $p=0.01$ ), emergency presentation (HR $1.82(p<0.01)$ and age (HR 1.06 per age bracket, $p$ $<0.01$ ) were adverse prognostic features and female sex (HR 0.82, $p$ $=0.04$ ) a good prognostic factor, the choice of R-CHOP or $\mathrm{R}$-miniCHOP had no influence over OS (R-CHOP reference, HR 0.95, $95 \% \mathrm{Cl} 0.73-1.22, p=0.68)$.

Non-anthracycline-containing regimens. Three-year OS was $52 \%$ (95\% Cl 44-59\%) for non-anthracycline-containing regimens ( $\mathrm{R}-$ CEOP $(n=166)$, R-GCVP $(n=37)$, total $n=203)$ (Fig. 5Sa), with numerically higher OS in those $65-79$ years $(54 \% ; 95 \% \mathrm{Cl} 44-63 \%)$ compared to $\geq 80$ years ( $49 \% ; 95 \% \mathrm{Cl} 38-60 \%)(p=0.42)$ (Fig. 5Sb). In this small subgroup 3-year OS after routine GP referral was $64 \%$ (95\% Cl $48-77 \%)$, NHS urgent cancer referral was $55 \%(95 \% \mathrm{Cl}$ $42-66 \%)$, and OS after emergency presentation was $39 \%(95 \% \mathrm{Cl}$ 26-51\%) (Fig. 5Sc). The 3-year OS for the 553 R-CVP-treated patients was $43 \%(95 \% \mathrm{Cl} 38-47 \%)$ and for 166 R-CEOP treated patients was $54 \%$ (46-52\%) (Fig. 6Sa, b). When the 325 patients receiving $\mathrm{R}$-CVP only with no hybrid regimens included were analysed specifically, the 3-year OS was equivalent (3-year OS $44.1 \%$ (95\% Cl 38.9-50.0\%). 
Table 3. Baseline characteristics in patients 80 years and over according to dose intensity of R-CHOP.

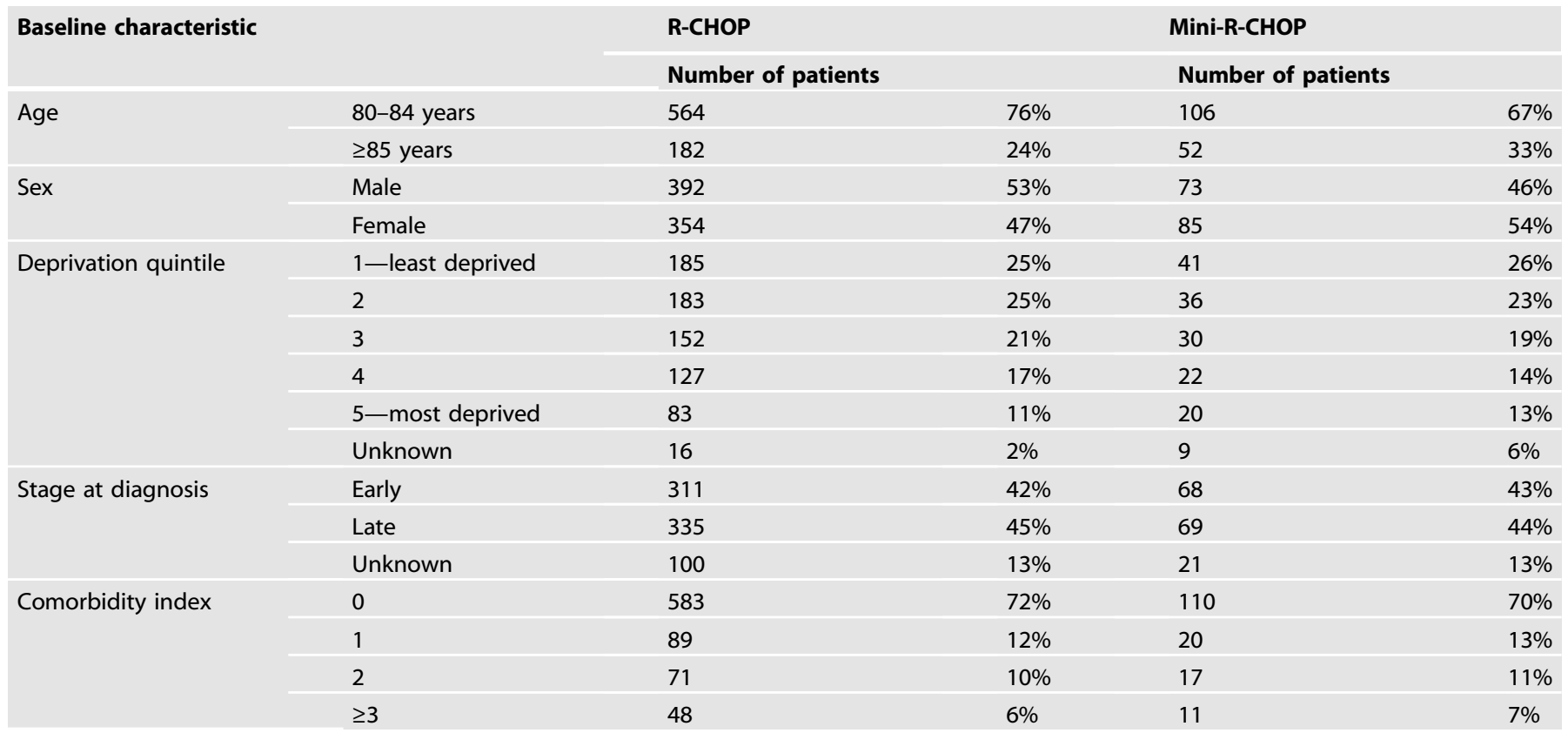

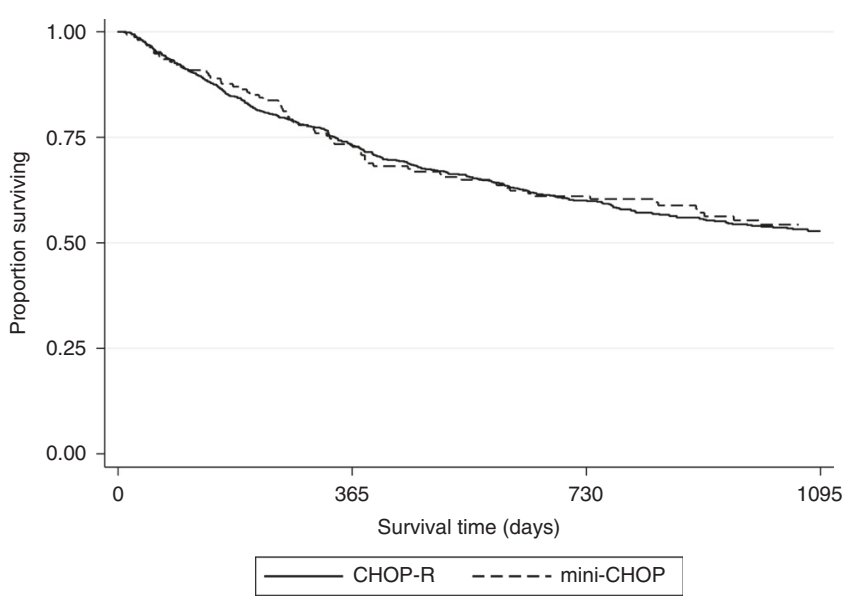

Fig. 5 OS for patients aged $\geq 80$ years, treated with either R-CHOP or R-mini-CHOP.

\section{DISCUSSION}

We present the analysis of a large real-world DLBCL dataset of an elderly ( $\geq 65$ years) DLBCL population diagnosed over a three-year consecutive period (2013-2015). We report four key findings that we believe are generalisable to elderly $D L B C L$ patients. Firstly, to our knowledge, the DLBCL survival outcomes presented are the first to describe in an adjusted analysis, that timed diagnostic NHS urgent cancer referral pathway (rapid early haemato-oncology access within 14-days) produced the most superior overall and lymphoma-specific survival. Secondly, patients presenting as an emergency at diagnosis have an inferior overall and lymphomaspecific survival on MVA when compared to standard non-2 week wait GP referrals. Thirdly, patients $\geq 80$ years have equivalent OS whether they receive R-CHOP or R-miniCHOP. Fourthly, elderly males have the worst OS of all groups studied.

Since the NHS timed urgent cancer referral pathway was implemented in the year 2000, analyses demonstrating its effectiveness in improving survival across solid cancers have been mixed. Some solid cancer series have suggested little benefit $[16,17]$ although a large meta-analysis demonstrated worse outcomes where diagnosis and treatment are delayed [18]. A small study in DLBCL did not show that time-to-diagnosis influenced OS [19]. In the first comprehensive nationwide analysis of its kind in DLBCL, we report the most superior outcomes were recorded for 1635 R-CHOP-treated patients presenting on a timed NHS urgent cancer referral pathway via "the Two Week Wait" where patients' investigations and diagnosis are expedited as quickly as possible. The pathway mandates that patients see a specialist within 14 days and enter treatment within 62 days of initial primary care referral. Our data suggest that this pathway reduces risk of mortality in $\mathrm{R}-\mathrm{CHOP}$ treated patients compared with standard GP referrals. These findings in part validate this strategy in enhancing DLBCL patient outcomes. DLBCL is a proliferative and aggressive disease where rapid diagnostic pathways make clinical sense. Our findings also suggest that, in order to improve future outcomes, reducing the proportion of patients presenting as an emergency is desirable. Emergency presentation had the lowest recorded survival and was also the most commonly observed diagnostic route (34\%). This may in part be due to patients not presenting with early detectable signs or symptoms, or a failure to recognise signs and symptoms of lymphoma although this simply may represent aggressive disease biology with adverse clinical features in some patients. We cannot be certain that better education will improve outcomes for all patients, particularly those who present with a clinical presentation requiring emergency care. However, to improve early recognition, national patient associations (e.g. Lymphoma Action, Blood (ancer UK) have developed tool kits designed to aid primary care practitioners' knowledge in recognition of the early presenting signs and symptoms of the disease, which may be subtle and not always immediately clinically apparent. Whether the NHS urgent cancer referral pathway results in improved OS in other haematological cancers remains an open, unexplored question.

There is a further drive by NHS England to diagnose cancer as early as possible [20] (2019 NHS plan), by raising symptom awareness, and implementing faster diagnosis standards through the roll out of rapid diagnostic centres. Moreover, recent UK population-based analysis have also described the potential impact of COVID19-related disruption to the NHS urgent cancer 
pathway and the potential negative impact on long term OS this may have [21, 22].

Recent data $[23,24]$ has clearly demonstrated that diagnosis-totreatment interval (DTI) in DLBCL and other aggressive NHL has an inverse effect on event-free survival. Our data has validated this in a population-based setting and also suggests that shorter DTI is more prevalent in patients presenting as an emergency as might be expected. The short time-to-diagnosis that the NHS urgent cancer pathway strategy enables provides decision-making control for the patient and physician regarding pace of DTI.

In our series, elderly males demonstrated the poorest OS (3-year OS 51\%). This finding mirrors published clinical trial data [25-27] and non-trial data [28] where confirmatory large series of studies demonstrated that elderly men achieve inferior outcomes when compared to their age-matched female counterparts. Recently, the HOVON-84 randomised clinical trial demonstrated that increased intensification of rituximab dosing early in treatment alongside CHOP-14 did not improve outcomes in DLBCL patients between 18 and 80 years compared to those receiving R-CHOP-14 [29]. Pharmacokinetic data has demonstrated enhanced clearance of the anti-CD20 in elderly males [25]. Whether enhancing rituximab dosage in trials specifically powered to assess in elderly patients remains unanswered.

Our data also demonstrate inferior outcomes for patients who present with pre-existing medical co-morbidities; in line with previous large population studies of DLBCL[30] in other countries. A similar outcome is not observed for lymphoma-specific survival; a possible consequence of competing mortalities resulting from significant co-morbidities.

The most common regimen choice was R-CHOP ( $48 \%$ of all cases, $80.5 \%$ of known front-line regimen) with recorded outcomes consistent with population studies from the US [31] and Sweden [32]. Approaches to enhance these outcomes have proven difficult in the front-line setting. Recent studies have attempted to improve outcomes by the addition of novel agents to the R-CHOP backbone. For example, the addition of ibrutinib to $\mathrm{R}-\mathrm{CHOP}$ enhanced efficacy in patients $<60$ years but was associated with enhanced toxicity in those $>60$ years [14].

Many elderly patients are not suitable for full dose intensity R-CHOP. Options exist for these patients are undoubtedly influenced by performance status, and the presence of preexisting comorbid conditions. Such patients often receive dose attenuated regimens such as R-miniCHOP [5]. Our series contained 313 patients treated with R-miniCHOP and reported an almost identical two-year OS of $60 \%$ in those $\geq 80$ years as reported in the LYSA trial. Three-year OS of $54 \%$ confirmed its efficacy across a broader real-world population. We also report 155 patients in the 65-79-year group unable to receive full dose intensity R-CHOP but who received R-miniCHOP. Three-year OS of $59 \%$ suggests younger patients may derive considerable benefit from dose attenuated regimens when they are otherwise clinically unsuitable to receive standard $\mathrm{R}-\mathrm{CHOP}$ due to frailty or dose-limiting comorbidities. We also report a similar 3 -year OS of $54 \%$ in a large R-CEOP cohort and recognise this option is a valid alternative approach in these patients who may otherwise be considered for R-miniCHOP due to frailty or cardiac comorbidity.

A further area of uncertainty is the importance of dose intensity of $\mathrm{R}$-CHOP in those $\geq 80$ years. There is a theoretical risk of inferiority of dose attenuation when compared to full dose $\mathrm{R}-\mathrm{CHOP}$ as several small studies have documented that retaining dose intensity improves outcomes in younger patients [33-36]. However, in the largest series analysed to date, we demonstrate that survival outcomes for R-CHOP $(n=746)$ and R-mini-CHOP ( $n$ $=158$ ) treated patients $\geq 80$ years were superimposable, confirming the equivalence of R-mini-CHOP to higher dose intensity $\mathrm{R}-\mathrm{CHOP}$ in this subgroup. We do recognise that patients included in the $\mathrm{R}-\mathrm{CHOP}$ group may have received some degree of dose attenuation between full and R-CHOP doses and the data should therefore be interpreted in light of this.

Our study has several limitations. It would have been desirable to have more complete data including full IPI components and other factors known to be associated with an inferior outcome in DLBCL. It remains possible that unmeasured confounders may have influenced results. For example, patients presenting as an emergency could have a higher proportion of extranodal disease sites and more bulky disease; factors known to be associated with inferior outcome. Based on our results, we cannot state definitively if the NHS urgent cancer pathway strategy helps patients with these additional factors or whether inferior disease biology specifically is the primary driver of this presentation in these patients. We do however note that early-stage patients presenting as an emergency have an inferior OS than all other groups other than late-stage emergency presentations, which suggests that multiple extranodal nodal sites or overall disease burden may not be the only driver to worse outcomes seen.

It would also be desirable to present a more granular analysis of relative dose intensity. Despite this, large recent studies show that relative dose intensity correlates very closely with intended dose intensity, with intended dose intensity the significant driving factor associated with survival [9]. We also recognise the lack of specific comorbidity classifications e.g. cardiac and lack of prognostic biological data (e.g. cell of origin, MYC/BCL2 status). These factors may have minimised the effect of unmeasured potential confounding variables.

It is possible that the lymphoma-specific survival rates observed in this cohort are higher than those previously observed in equivalent national studies [7] due to under recording of DLBCL as a specific cause of death. Care should be taken when comparing lymphoma-specific survival within our cohort to other studies outside the UK as procedures for recording causes of death on the death certificate, and the analytic methods used could vary. Nevertheless, outcomes within the MVA are consistent with the OS analysis and are clinically relevant.

We also recognise that the overall recording of comorbidities is relatively low for patients within ages studied. This may be attributable to low numbers of comorbid illnesses being recorded during inpatient episodes and the inability to record co-morbid illnesses in all patients treated in a non-inpatient setting. We recognise that national, systematic comorbidity data collection methods are needed to ensure all comorbidities are recorded across the hospital episodes and primary care. Further efforts to improve the availability of primary care data to capture such comorbidity data will provide more information going forward to optimise future analyses.

Finally, we note that $40 \%$ of patients in the total dataset had no treatment information recorded or had another treatment outside those analysed. Overall, these patients had an inferior survival to those patients with treatment detailed in the manuscript. This, therefore, represents a large, unexplored cohort within the national registry. We recognise the importance of high-quality palliative care and palliative therapies in this setting but acknowledge that a detailed analysis of these patients in outside the scope of this manuscript.

\section{CONCLUSION}

We have demonstrated that patients who are referred via the timed '2-week wait' NHS urgent cancer referral pathway where initial investigations are performed within 14 days achieved the best documented survival outcomes. We also confirm the efficacy of the R-miniCHOP regimen based on intended dose intensity data in patients $\geq 80$ years and its probable equivalence to $\mathrm{R}-\mathrm{CHOP}$ in a real-world setting. In our patient cohort, we have observed that elderly patients over 80 years have significantly inferior 
outcomes-in particular elderly males. This necessitates further research into causation of this gender discrepancy and the use of novel non-chemotherapeutic targeted agents to improve outcomes in elderly and frail DLBCL patients.

\section{DATA AVAILABILITY}

For data sharing and availability, please contact the corresponding author, Prof. Paul Fields.

\section{REFERENCES}

1. Flowers CR, Sinha R, Vose JM. Improving outcomes for patients with diffuse large B-cell lymphoma. CA Cancer J Clin. 2010;60:393-408.

2. Morgan G, Vornanen M, Puitinen J, Naukkarinen A, Brincker $H$, Olsen J, et al. Changing trends in the incidence of non-Hodgkin's lymphoma in Europe. Biomed Study Group. Ann Oncol. 1997;8(Suppl 2):49-54.

3. Morton LM, Wang SS, Devesa SS, Hartge P, Weisenburger DD, Linet MS. Lymphoma incidence patterns by WHO subtype in the United States, 1992-2001. Blood. 2006;107:265-76.

4. NHS Executive. Cancer waiting times: achieving the two week target: Health service circular 1999. 1999/205.

5. Peyrade F, Jardin F, Thieblemont C, Thyss A, Emile JF, Castaigne S, et al. Attenuated immunochemotherapy regimen (R-miniCHOP) in elderly patients older than 80 years with diffuse large B-cell lymphoma: a multicentre, single-arm, phase 2 trial. Lancet Oncol. 2011;12:460-8.

6. Peyrade F, Bologna S, Delwail V, Emile JF, Pascal L, Ferme C, et al. Combination of ofatumumab and reduced-dose CHOP for diffuse large B-cell lymphomas in patients aged 80 years or older: an open-label, multicentre, single-arm, phase 2 trial from the LYSA group. Lancet Haematol. 2017;4:46-55.

7. Juul MB, Jensen PH, Engberg $H$, Wehberg $S$, Dessau-Arp A, Haziri D, et al. Treatment strategies and outcomes in diffuse large B-cell lymphoma among 1011 patients aged 75 years or older: a Danish population-based cohort study. Eur J Cancer. 2018:99:86-96.

8. Eyre TA, Salisbury R, Eyre DW, Watson C, Collins GP, Hatton CS. Results of a large retrospective analysis of the effect of intended dose intensity of R-CHOP on outcome in a cohort of consecutive, unselected elderly patients with de novo diffuse large B cell lymphoma. Br J Haematol. 2016;173:487-91.

9. Eyre TA, Martinez-Calle N, Hildyard C, Eyre DW, Plaschkes H, Griffith J, et al. Impact of intended and relative dose intensity of R-CHOP in a large, consecutive cohort of elderly diffuse large B-cell lymphoma patients treated with curative intent: no difference in cumulative incidence of relapse comparing patients by age. J Intern Med. 2019;285:681-92

10. Oberic L, Peyrade F, Puyade M, Bonnet C, Dartigues-Cuilleres P, Fabiani B, et al. Subcutaneous Rituximab-MiniCHOP compared with subcutaneous RituximabMiniCHOP plus lenalidomide in diffuse large B-cell lymphoma for patients age 80 years or older. J Clin Oncol. 2021. https://doi.org/10.1200/JCO.20.02666.

11. Bright CJ, Lawton S, Benson S, Bomb M, Dodwell $S$, Henson $K E$, et al. Data resource profile: the systemic anti-cancer therapy (SACT) dataset. Int J Epidemiol. 2020;1:15-15l.

12. Elliss-Brookes L, McPhail S, Ives A, Greenslade M, Shelton J, Hiom S, et al. Routes to diagnosis for cancer - determining the patient journey using multiple routine data sets. Br J Cancer. 2012;107:1220-6.

13. Phillips E, Kirkwood A, Barrans S, Burton C, Collins G, Davies A, et al. Inotuzumab ozogamicin plus R-CVP is a feasible and effective regimen for frontline treatment of dlbcl in patients who are unfit for R-CHOP: results of a randomised phase 2 UK NCRI trial. Hemasphere. 2020;4:75.

14. Younes $A$, Sehn LH, Johnson $\mathrm{P}$, Zinzani PL, Hong $\mathrm{X}$, Zhu J, et al. Randomized phase III trial of ibrutinib and rituximab plus cyclophosphamide, doxorubicin, vincristine, and prednisone in non-germinal center B-cell diffuse large B-cell lymphoma. J Clin Oncol. 2019;37:1285-95.

15. Davies A, Cummin TE, Barrans S, Maishman T, Mamot C, Novak U, et al. Geneexpression profiling of bortezomib added to standard chemoimmunotherapy for diffuse large B-cell lymphoma (REMoDL-B): an open-label, randomised, phase 3 trial. Lancet Oncol. 2019;20:649-62.

16. Potter S, Govindarajulu S, Shere M, Braddon F, Curran G, Greenwood R, et al. Referral patterns, cancer diagnoses, and waiting times after introduction of two week wait rule for breast cancer: prospective cohort study. BMJ. 2007;335:288.

17. Aslam MI, Chaudhri S, Singh B, Jameson JS. The "two-week wait" referral pathway is not associated with improved survival for patients with colorectal cancer. Int J Surg. 2017;43:181-5.

18. Hanna TP, King WD, Thibodeau $S$, Jalink M, Paulin GA, Harvey-Jones $E$, et al. Mortality due to cancer treatment delay: systematic review and meta-analysis. BMJ. 2020:371:4087.
19. Nikonova A, Buckstein R, Giurgis H, Cheung MC. Predictors of delay in diagnosis and treatment in diffuse large B-cell lymphoma and impact on survival. Blood. 2013;122:4322.

20. NHS plan 2019: Chapter 3 Care quality and outcomes: 55-62.

21. Sud A, Torr B, Jones ME, Broggio J, Scott S, Loveday C, et al. Effect of delays in the 2-week-wait cancer referral pathway during the COVID-19 pandemic on cancer survival in the UK: a modelling study. Lancet Oncol. 2020;21:1035-44.

22. Sud A, Jones ME, Broggio J, Loveday C, Torr B, Garrett A, et al. Collateral damage: the impact on outcomes from cancer surgery of the COVID-19 pandemic. Ann Oncol. 2020;31:1065-74.

23. Maurer MJ, Ghesquieres $\mathrm{H}$, Link BK, Jais JP, Habermann TM, Thompson CA, et al. Diagnosis-to-treatment interval is an important Clinical factor in newly diagnosed diffuse large B-cell lymphoma and has implication for bias in clinical trials. J Clin Oncol. 2018;36:1603-10.

24. Olszewski AJ, Ollila T, Reagan JL. Time to treatment is an independent prognostic factor in aggressive non-Hodgkin lymphomas. Br J Haematol. 2018;181:495-504.

25. Pfreundschuh M, Muller C, Zeynalova S, Kuhnt E, Wiesen $M H$, Held G, et al. Suboptimal dosing of rituximab in male and female patients with DLBCL. Blood. 2014;123:640-6.

26. Riihijarvi S, Taskinen $M$, Jerkeman $M$, Leppa $S$. Male gender is an adverse prognostic factor in B-cell lymphoma patients treated with immunochemotherapy. Eur J Haematol. 2011;86:124-8.

27. Carella AM, de Souza CA, Luminari S, Marcheselli L, Chiappella A, di Rocco A, et al. Prognostic role of gender in diffuse large B-cell lymphoma treated with rituximab containing regimens: a Fondazione Italiana Linfomi/Grupo de Estudos em Molestias Onco-Hematologicas retrospective study. Leuk Lymphoma. 2013;54:53-57.

28. Eyre TA, Martinez-Calle N, Hildyard C, Eyre DW, Plaschkes H, Griffith J, et al. Male gender is an independent predictor for worse survival and relapse in a large, consecutive cohort of elderly DLBCL patients treated with R-CHOP. Br J Haematol. 2019;186:94-98.

29. Lugtenburg PJ, de Nully Brown $P$, van der Holt B, D'Amore FA, Koene HR, de Jongh $E$, et al. Rituximab-CHOP with early rituximab intensification for diffuse large B-cell lymphoma: a randomized phase III trial of the HOVON and the Nordic lymphoma group (HOVON-84). J Clin Oncol. 2020;38(29):3377-87.

30. Hershman DL, McBride RB, Eisenberger A, Tsai WY, Grann VR, Jacobson JSDoxorubicin. cardiac risk factors, and cardiac toxicity in elderly patients with diffuse B-cell non-Hodgkin's lymphoma. J Clin Oncol. 2008;26:3159-65.

31. Giri U, Martin MG. Survival outcomes in the very elderly with $D L B C L$ prior to and after the introduction of rituximab: a US population-based study. Blood Adv. 2017;1:615-8.

32. Hedstrom G, Hagberg O, Jerkeman M, Enblad G, Swedish Lymphoma Study G. The impact of age on survival of diffuse large B-cell lymphoma-a populationbased study. Acta Oncol. 2015;54:916-23.

33. Terada $Y$, Nakamae $H$, Aimoto $R$, Kanashima $H$, Sakamoto $E$, Aimoto $M$, et al. Impact of relative dose intensity (RDI) in CHOP combined with rituximab (RCHOP) on survival in diffuse large B-cell lymphoma. J Exp Clin Cancer Res. 2009;28:116.

34. Hirakawa T, Yamaguchi H, Yokose N, Gomi S, Inokuchi K, Dan K. Importance of maintaining the relative dose intensity of CHOP-like regimens combined with rituximab in patients with diffuse large B-cell lymphoma. Ann Hematol. 2010;89:897-904.

35. Vidal $L$, Lando $S$, Vaxman I, Shochat $T$, Raanani $P$, Gurion $R$, et al. The effect of $\mathrm{R}-\mathrm{CHOP}$ dose reduction on overall survival of elderly patients with $\mathrm{DLBCL}-$ comparative study. Leuk Lymphoma. 2018;59:904-10.

36. Kanemasa $Y$, Shimoyama T, Sasaki Y, Tamura M, Sawada T, Omuro Y, et al. The impacts of initial and relative dose intensity of R-CHOP on outcomes of elderly patients with diffuse large B-cell lymphoma. Leuk Lymphoma. 2017;58:736-9.

\section{ACKNOWLEDGEMENTS}

TE acknowledge support by the National Institute for Health Research (NIHR) Oxford Biomedical Research Centre Programme. KA acknowledge support by the National Institute for Health Research (NIHR) UCLH Biomedical Research Centre Programme. The views expressed are those of the authors and not necessarily those of the funding bodies.

\section{AUTHOR CONTRIBUTIONS}

PAF and LH designed the study. PAF, TE and LH co-wrote the manuscript, which all authors critically reviewed. LH extracted data and performed the statistical analysis. 


\section{FUNDING}

Lymphoma Research Trust.

\section{ETHICS APPROVAL AND CONSENT TO PARTICIPATE}

Data were obtained from PHE NCRAS via a formal application to the organisation's PHE's Office for Data Release (ODR). Data were assessed as suitable for release without specific ethical permissions by ODR (ODR assignment no:1617_136) and the NCRAS Caldicott Guardian. The study was performed in accordance with the Declaration of Helsinki.

\section{CONSENT TO PUBLISH}

Not applicable.

\section{COMPETING INTERESTS}

The authors declare no competing interests.

\section{ADDITIONAL INFORMATION}

Supplementary information The online version contains supplementary material available at https://doi.org/10.1038/s41416-021-01525-4.

Correspondence and requests for materials should be addressed to P.A. Fields
Reprints and permission information is available at http://www.nature.com/ reprints

Publisher's note Springer Nature remains neutral with regard to jurisdictional claims in published maps and institutional affiliations.

Open Access This article is licensed under a Creative Commons Attribution 4.0 International License, which permits use, sharing, adaptation, distribution and reproduction in any medium or format, as long as you give appropriate credit to the original author(s) and the source, provide a link to the Creative Commons license, and indicate if changes were made. The images or other third party material in this article are included in the article's Creative Commons license, unless indicated otherwise in a credit line to the material. If material is not included in the article's Creative Commons license and your intended use is not permitted by statutory regulation or exceeds the permitted use, you will need to obtain permission directly from the copyright holder. To view a copy of this license, visit http://creativecommons. org/licenses/by/4.0/.

(c) The Author(s) 2021 